\title{
Role of CT in Evaluation of Blunt Abdominal Trauma
}

\author{
Adel Mohamed El Wakeel ${ }^{1}$, Rehab Mohamed Habib ${ }^{1}$, Abeer Nabil Ali ${ }^{2}$ \\ ${ }^{1}$ Radiodiagnosis Department, Faculty of Medicine, Menoufia University, Shibeen El koom, Egypt \\ ${ }^{2}$ Radiodiagnosis Department, Fever Hospital, Menoufia, Egypt
}

Email address:

Abeerarafa85@yahoo.com (A. N. Ali)

\section{To cite this article:}

Adel Mohamed El Wakeel, Rehab Mohamed Habib, Abeer Nabil Ali. Role of CT in Evaluation of Blunt Abdominal Trauma. International Journal of Medical Imaging. Vol. 3, No. 5, 2015, pp. 89-93. doi: 10.11648/j.ijmi.20150305.11

\begin{abstract}
CT was regard as golden standard method for evaluation of blunt abdominal trauma. CT allow good detection of any organ injury and determine its grade upon which the management will be performed. The aim of our study is to evaluate the role of CT in blunt abdominal trauma. This study included 50 patients presented with blunt abdominal trauma. Patients were subjected to clinical history and examination followed by MDCT scan with IV contrast. The results of our study proved that the liver was the most common injured organ representing $40 \%$, spleen $30 \%$, kidney $16 \%$, peritoneum $8 \%$, while the pancreas was the least common one representing only $2 \%$ of all our cases .Grade II hepatic injury was the most common representing $65 \%$ of patients with hepatic injury, also grade II splenic injury was the most common representing $73 \%$ of patients with splenic injury. Both grade I \& II renal injuries were the most common each representing $37.5 \%$. In our study 3 cases $(6 \%)$ were detected having organ injury associated with active bleeding. We conclude that MDCT is the gold standard method for evaluation of blunt abdominal trauma as it play an important role not only in detection of organ injury, but also in the grading of this injury on which the management will be performed. Also it is important in the follow up of cases which undergo conservative treatment. In addition MDCT proved to be highly sensitive in detection of active hemorrhage which is a life threatening condition.
\end{abstract}

Keywords: Computed Tomography, Blunt Trauma, Abdomen

\section{Introduction}

Trauma is the leading cause of death under the age of forty and of all traumatic deaths, $10 \%$ belong to abdominal injuries .Computed tomography (CT) scan is the gold standard in abdominal blunt trauma in stable patients because it reduces the number of laparotomies ${ }^{(1)}$.

Hemodynamically stable patients with blunt trauma and suspected abdominal vascular injuries may benefit from abdominal CT scanning, which helps to localize a hematoma and evaluate solid organ injuries ${ }^{(2,3,4)}$.

CT scan has $100 \%$ sensibility for hemoperitoneum and contrast enhanced sequence allows the detection of active bleeding followed by embolization or surgery ${ }^{(5,6)}$.

The role of the radiologist is to look for the following aspects that are to be expected in abdominal blunt trauma: hemoperitoneum, contrast blush consistent with active bleeding, lacerations, contusions, parenchymal hematomas, subcapsular hematomas, devascularization of organs or part of organs, free intraperitoneal or retroperitoneal air, diaphragmatic rupture ${ }^{(l)}$.
Statistically, the majority of blunt abdominal trauma is caused by car accidents (more than $75 \%$ of cases) followed by aggressions ( $15 \%$ of cases), accidental falls (6 to 9\%) and child abuse ${ }^{(7)}$.

The most common injured organ in blunt abdominal trauma is the spleen. In $50 \%$ of cases, it is the only intraperitoneal injured organ ${ }^{(1,8,9)}$.

Although the spleen is relatively protected under the ribcage, injury due to rapid deceleration, such as occurs in motor vehicle crashes, direct blows to the abdomen in domestic violence, or leisure and play activities such as bicycling, frequently result in a variety of splenic injuries. Also there have been case reports of splenic injury following colonoscopy $^{(10)}$.

The liver is the second most frequent intraperitoneal solid organ in adults and the first one in children ${ }^{(7)}$.

Kidneys are frequently injured in abdominal trauma (up to $80-90 \%$ of cases) but the majority of them are managed nonoperatively $(95 \%)^{(1,11)}$.

The pancreas, sitting in a relatively protected position high in the retroperitoneum, is infrequently injured in typical blunt 
injuries (eg, from motor vehicle crashes) compared with its splenic and hepatic counterparts.Accordingly, many blunt pancreatic injuries are not immediately recognized and consequently end up causing higher morbidity and mortality rates than observed in injuries to other intraperitoneal organs (12)

Aim of the work:

The aim of the study is to evaluate the role of CT in blunt abdominal trauma.

\section{Materials and Methods}

This study was conducted in the period from July 2014 to April 2015 at emergency department of both Menoufia University Hospital \& Shibeen El Koom Teaching Hospital. All patients presented with blunt abdominal trauma. Patients with positive $\mathrm{CT}$ findings were selected in the study. This study included 50 patients (36 males \& 14 females) with different age groups, all 50 patients were subjected to full clinical history and examination \& multi-detector CT (MDCT) scan.

CT abdomen was performed at the CT unit of the emergency department using the CT scanner (multi-detector 4).

Protocol of the CT scan of the abdomen \& pelvis:

- Scan parameters 100 - $120 \mathrm{KVP}, 200$ - 300 mAS.

- Slice thickness was 3-5 mm.

- The scan was done perpendicular to the sagittal plane of the patient in supine position and started by taking some basal chest cuts (looking for accompanying chest injury) down to the level of symphysis pubis.

- The conscious patients were instructed not to move during scan to avoid motion artifacts.

- Pre-contrast scan was done to all patients followed by post-contrast scan. Patients received 100-150 ml of contrast agent (Iopromide $370 \mathrm{mg} / \mathrm{ml}$ ) which was given intravenously by means of automated injector at an injection rate of $3.5 \mathrm{ml} / \mathrm{sec}$. Antecubital vein is used as the access route of 18-gauge intravenous needle. For children (the dose was $1.5-2 \mathrm{~mL} / \mathrm{kg}$ ) were injected at 3$3.5 \mathrm{ml} / \mathrm{second}$. Abdominal scanning was done at 70 seconds after contrast administration.

- Axial images were obtained with window width around 65 and window level around 120.

- Delayed scan was done for three patients with renal injury for assessment of collecting system involvement. The scan was done at 5 minutes after contrast administration.

The radiological signs looked for are:

- Fluid collection within the different pouches and its amount (mild, moderate or marked).

- Any internal organ injury and its degree and if it is isolated organ injury or it is multiorgan injury.

- Signs of active bleeding (extravasation of contrast material).

- Vascular injuries.

- Retroperitoneal hematoma.

\section{Results}

Car accidents represented the most common cause (70\%) and more common in males $(48 \%)$. The liver was the most common injured organ representing $40 \%$, spleen $30 \%$, kidneys $16 \%$, peritoneum $8 \%$, while the pancreas was the least common one representing only $2 \%$ of all our cases. In relation to age, the liver was the most common injured organ in children and young adults representing $65 \%$ of patients with liver injury, while the spleen was the most common injured one in adults representing $53.7 \%$ of the splenic injured patients (table 1). Grade II hepatic injury was the most common representing $65 \%$ of patients with hepatic injury (table 2). Also grade II splenic injury was the most common representing $73 \%$ of patients with splenic injury (figure1). Both grade I \& II renal injury were the most common each representing $37.5 \%$. In our study 3 cases $(6 \%)$ were detected having organ injury associated with active bleeding (figure 2).

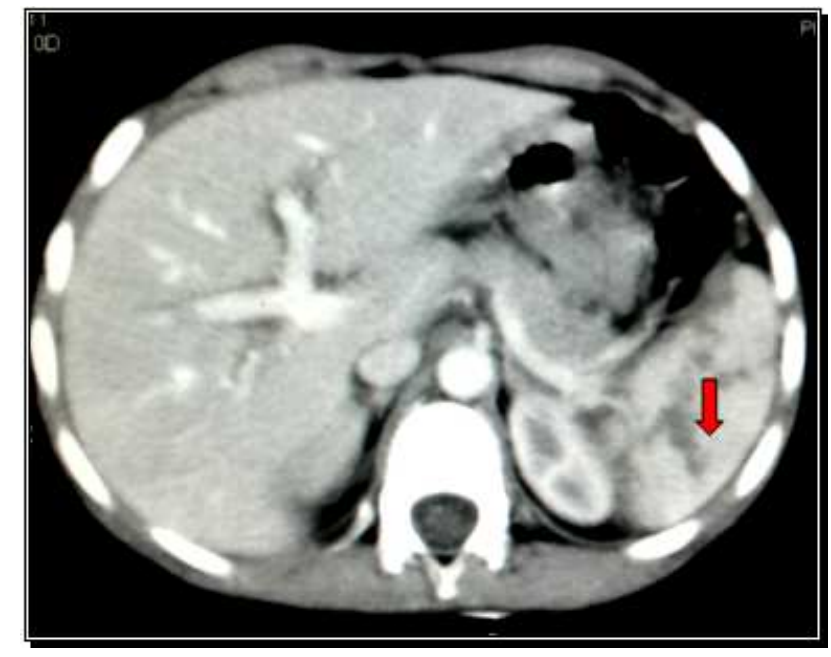

(A)

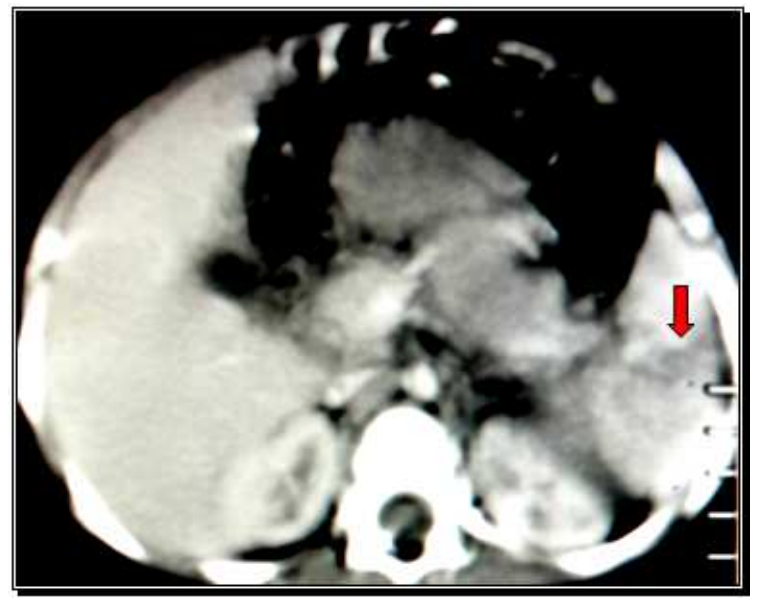

(B)

Figure 1. A 23 years old male presented with blunt abdominal trauma caused by motorcycle accident. Post-contrast CT scan of the abdomen axial cuts shows multiple small linear hypo-attenuation areas (red arrows) each about $3 \mathrm{~cm}$ in depth representing splenic lacerations (fig. 1 A,B). Grade II splenic injury. 


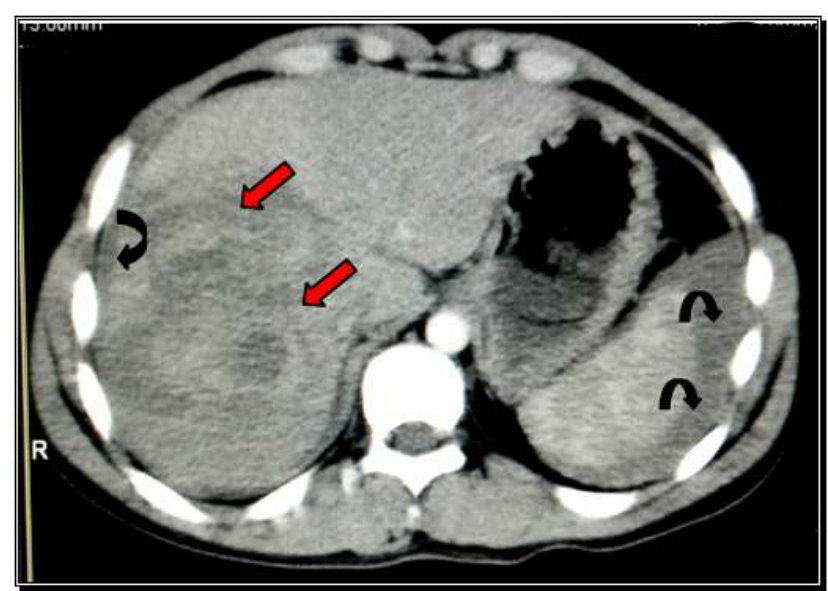

(A)

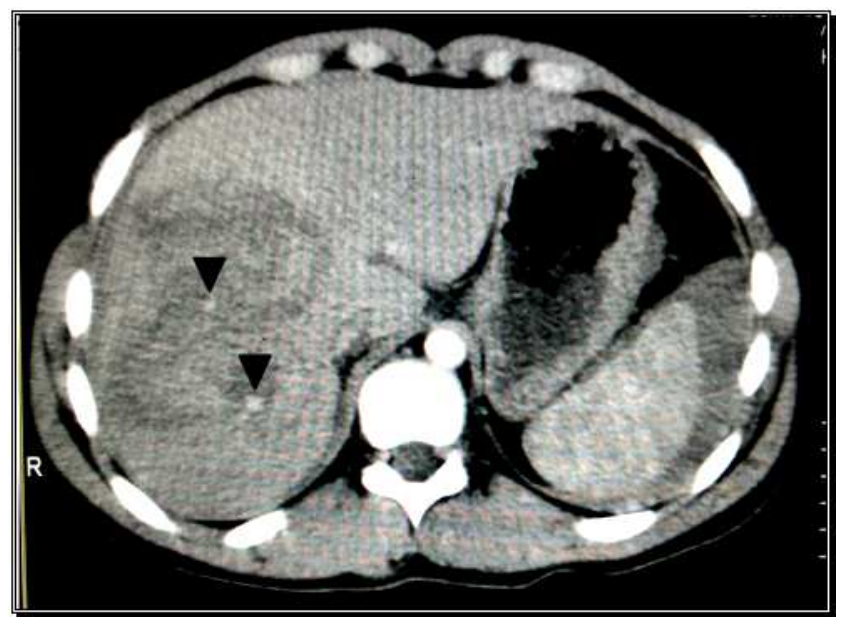

(B)

Figure 2. A 22 years old male presented with blunt abdominal trauma caused by falling from hight. Post-contrast CT scan of the abdomen, axial cuts shows parenchymal hematoma in the right hepatic lobe measuring $8 \times 9 \mathrm{~cm}$ (red arrows) (fig.2A). Note the high-attenuation arterial extravasation (arrow heads) within the hematoma representing active hemorrhage (fig.2B). Also there are peri-hepatic \& peri-splenic blood collection (curved black arrows) (fig.2A) . Grade II hepatic injury.

Table 1. The relation between the injured organ and age.

\begin{tabular}{llllll}
\hline \multirow{2}{*}{$\begin{array}{l}\text { The injured } \\
\text { organ }\end{array}$} & Total & \multicolumn{2}{l}{$\begin{array}{l}\text { Children \& young } \\
\text { adult(up to 20y) }\end{array}$} & \multicolumn{2}{l}{$\begin{array}{l}\text { Adult (More than } \\
\mathbf{2 0 y})\end{array}$} \\
\cline { 3 - 6 } & & $\mathbf{N}$ & $\mathbf{\%}$ & $\mathbf{N}$ & $\%$ \\
\hline Liver & 20 & 13 & 65 & 7 & 35 \\
Spleen & 15 & 7 & 46.7 & 8 & 53.3 \\
Kidney & 8 & 4 & 50 & 4 & 50 \\
Peritoneum & 4 & 2 & 50 & 2 & 50 \\
Pancreas & 1 & 100 & 0 & 0 & 0 \\
Combined & 2 & 1 & 50 & 1 & 50 \\
\hline
\end{tabular}

Table 2. Number and percentage of cases according to grades of liver injury .

\begin{tabular}{lll}
\hline Grade & $\mathbf{N}$ & $\mathbf{\% ( T = 2 0 )}$ \\
\hline G I & 0 & 0 \\
G II & 13 & 65 \\
G III & 3 & 15 \\
G IV & 3 & 15 \\
G V & 1 & 5 \\
G VI & 0 & 0 \\
\hline
\end{tabular}

\section{Discussion}

Trauma is one of the leading causes of death all over the world .The abdomen is one of the most common sites of the body vulnerable to be injured. The care of the trauma patient is demanding and requires speed and efficiency ${ }^{(13)}$.

CT has become the definitive imaging modality of choice when intra-abdominal injury is suspected. CT is rapid and highly sensitive and specific for many important injury types. The informations provided by CT allow prognosis of injury and selective non-operative management in blunt trauma ${ }^{(14)}$. In our study 36 cases were males \& 14 were females, the age ranged between two and 70 years old. The majority of our patients belonged to the first decade and the least were patients within their fifth decade.

Maria Daniela Podeanu et al in their results of 327 patients with blunt abdominal trauma examined during 2012, 52 cases representing $15,9 \%$ had CT detectable intraabdominal lesions, 37 were males and 15 were females. Age ranged between two and 83 years old. The majority of patients belonged to the third decade and the least were patients within their first decade. Regarding the cause of trauma, they found that the car accidents were $79 \%$ of cases, followed by accidental falling $(13 \%){ }^{(15)}$. In the current study, Car accidents represented the most common cause (70\%), followed by motorcycle accidents (14\%). This agree with Visrutaratna $P$ who reported that the majority of blunt abdominal trauma is caused by car accidents (more than $75 \%$ of cases) ${ }^{(7)}$.

Jansen JO et al and Isenhour JL et al stated that the most common injured organ in blunt abdominal trauma is the spleen. In $50 \%$ of cases, it is the only intra-peritoneal injured organ ${ }^{(8,9)}$. Also Cahir JG reported that the spleen is the most commonly injured solid abdominal organ following blunt abdominal trauma ${ }^{(16)}$. But in our study the liver was the most commonly injured organ representing $40 \%$ of all our cases, this is because the majority of our cases belonged to the first decade.

Visrutaratna $P$ stated that the liver is the second most frequent intra-peritoneal solid organ in adults and the first one in children ${ }^{(7)}$. As shown in our study the liver was the most common injured organ in children and young adults representing $65 \%$ of patients with liver injury, while the spleen was the most common injured one in adult representing $53.7 \%$ of the splenic injured patients. Romano $L$ et al reported that isolated hepatic lesions are rare and in 77$90 \%$ of cases, lesions of other organs and viscera are involved (17). This was against our study in which the majority of cases were isolated organ injury (47\%), and as the liver was the most injuried organ in our study so the hepatic lesions were nearly almost isolated .

Kawashima $A$ et al stated that urinary tract injuries occur in $3 \%-10 \%$ of all abdominal trauma patients, the kidney being the most commonly injured organ. The vast majorities $(80 \%-90 \%)$ of cases are secondary to blunt abdominal trauma, and most significant renal trauma is associated with injury to other major organs. On the other hand, up to $95 \%$ 
$98 \%$ of isolated renal injuries are considered minor injuries and are managed nonsurgically because they usually heal spontaneously without complications (18). Lee YJ et al recommended selective use of 5-minute delayed CT of the abdomen and pelvis to rule out leakage of contrast enhanced urine if renal pedicle injury is found ${ }^{(19)}$. In the current study eight cases with renal injury were detected representing $16 \%$ of all cases, and as recommended by Lee YJ et al, delayed scan (after 5- minutes) was done for three cases with suspected collecting system involvement, two of them showed urinary contrast extravasation.

Harris $A C$ et al and West $O C$ reported that most renal injuries are of the minor types and include contusion, subcapsular or perinephric hematoma and superficial laceration $^{(11,20)}$.

As shown in our study the majority of cases with renal injury were of grade I \& grade II each representing $37.5 \%$ of the renal injured patients. Buccimazza I et al in their study proved that isolated blunt injuries to the pancreas are rare when compared with liver and splenic injuries. The estimated injury rate ranges from $0.2 \%$ to $1.3 \%$ in patients sustaining blunt trauma ${ }^{(2 I)}$.

As regarding to our study, only one case with pancreatic injury was detected reprsenting $2 \%$ of our cases .This case was 11 years old male with car accident. It was left sided package injury as it was associated with perinephric hematoma, fluid collection in lesser sac and during surgery bowel injury was detected. This agree with Gupta A et al and Linsenmaier $U$ et al who stated that pancreatic injuries are rare, occurring in around $2 \%$ of blunt trauma patients but may be associated with high morbidity and mortality. Pancreatic injuries are rarely isolated, and organ injuries most commonly associated ${ }^{(22,23)}$.

Hamdi Hameed Almaramhy on his study on seven patients over a period of 5 years; 5 males and 2 females with a mean age of 7 years (age range 5-12 years), reported three (42\%) patients had associated abdominal organ injuries. Associated injuries were splenic lacerations in two patients and uretropelvic disruption of left kidney in one patient. Peri-pancreatic fluid collection in MDCT was noted in 2 patients; fluid was in maximum amount in the lesser sac ${ }^{(24)}$. In the current study almost all patients with organ injuries were associated with hemo-peritonium, peri-hepatic or peri-splenic fluid collections, fluid in the lesser sac or even minimal amounts in the pelvis. This agree with Pikoulis E et al and Lubner M et al who stated that CT scan has $100 \%$ sensibility for hemoperitoneum and contrast enhanced sequence allows the detection of active bleeding followed by embolization or surgery ${ }^{(2,3)}$.

Jürgen K. Willmann et al showed that active hemorrhage originating from various organs including liver, spleen, pancreas, kidneys, bowel, mesentery, and abdominal soft tissues can be detected on CT. Also they stated that identification of active hemorrhage is of paramount importance because this finding may indicate a lifethreatening hemorrhage and has implications for proper emergency treatment ${ }^{(25)}$.
Jürgen K. Willmann et al noted that active hemorrhage in patients after blunt abdominal trauma is most frequently visible as a jet of extravasated contrast agent on MDCT. When extravasation is detected, immediate surgical or angiographic therapy is required ${ }^{(25)}$. In our study $6 \%$ of cases were detected having organ injury associated with active bleeding.

\section{Conclusions}

Multi-detector CT proved to be very sensitive to all types of organ injury. Also it considered the imaging modality of choice to diagnose the solid organ injury and determine the grading of this injury on which the management will be performed. In addition MDCT proved to be highly sensitive in detection of active hemorrhage which is a life threatening condition. Pre-contrast CT scan followed by post-contrast scan using IV contrast material should be done for all patients presented with blunt abdominal trauma whenever possible. Also delayed scan should be done for selected cases with renal injury for evaluation of collecting system involvement.

\section{References}

[1] Stephen Ledbetter and Robin Smithuis, ( 2007) ,Radiology Assistant, Acute Abdomen - Role of CT in Trauma. AJR , 189:1421-1427.

[2] Dayal M, Gamanagatti S, Kumar A. ( 2013 ) Imaging in renal trauma. World J. Radiol.;5(8):275-84.

[3] Genovese EA, Fonio P, Floridi C, et al. ( 2013 ) Abdominal vascular emergencies: US and CT assessment. Crit Ultrasound J.,5 Suppl 1:S10.

[4] Vu M, Anderson SW, Shah N, et al. ( 2010 ) CT of blunt abdominal and pelvic vascular injury. Emerg Radiol.;17(1):219.

[5] Pikoulis E, Kailidou E Katsiva V, et al. (2005) Contrast Enhanced Spiral CT Evaluation of Blunt Abdominal Trauma, JBR-BTR; 88: 61-65.

[6] Lubner M, Menias C, Rucker C, et al. (2007) Blood in the belly: CT findings of hemoperitoneum. Radiographics; 27 : 109-125.

[7] Visrutaratna P, Na-Chiangmai W. (2008) Computed tomography of blunt abdominal trauma in children. Singapore Med. J., 49: 352-358.

[8] Jansen JO, Yule SR, Udon MA. (2008) Investigation of blunt abdominal trauma, BMJ; 336: 938-942.

[9] Isenhour JL, Marx J. (2007) Advances in abdominal trauma. Emerg Med Clin N Am;25:713-733.

[10] Fishback SJ, Pickhardt PJ, Bhalla S, et al . (2011) Delayed presentation of splenic rupture following colonoscopy: clinical and CT findings. Emerg Radiol.; 18(6):539-44.

[11] Harris AC, Zwirewich CV, Lyburn ID, et al. (2001) CT Findings in Blunt Renal Trauma. RadioGraphics;21:201-214. 
[12] Ahmed N, Vernick JJ. (2009) Pancreatic injury. South Med. J.,102 (12):1253-6.

[13] John Udeani and John Geibel, ( 2008). Abdominal Trauma, Blunt. eMedicine, Specialties, Trauma, Abdominal Trauma.

[14] Nadia Mama, Hela Jemni, Nadia Arifa et al,(2012) Abdominal Trauma Imaging, http://dx.doi.org/10.5772/50426.

[15] Maria Daniela Podeanu , Andrada Treaba , Nina Sincu et al ( 2013) CT findings in patients with blunt abdominal trauma, AMT, v. II, (4)259-262.

[16] Cahir JG (2004).Multislice CT of the abdomen. BJR; 177:S64-S73.

[17] Romano L, Giovine S, Guidi G, et al ( 2004) Hepatic trauma: $\mathrm{CT}$ findings and considerations based on our experience in emergency diagnostic imaging. Eur. J. Radiol 50(1):59-66.

[18] Kawashima A, Sandler CM, Corl FM, et al.( 2001) Imaging of renal trauma: a comprehensive review. RadioGraphics;21(3):557-574.

[19] Lee YJ, Oh SN, Rha SE,et al ( 2007). Renal trauma. Radiol Clin North Am;45(3):581-592.
[20] West OC, (2000). Intraperitoneal Abdominal Injuries .American Roentgen Ray Society, 2000 : 87-98.

[21] Buccimazza I, Thomson SR, Anderson F, et al.( 2006). Isolated main pancreatic duct injuries. Spectrum and management. Am. J. Surg.,191:448-52.

[22] Gupta A, Stuhlfaut JW, Fleming KW, et al (2004). Blunt trauma of the pancreas and biliary tract: a multimodality imaging approach to diagnosis. Radiographics; 24(5):1381-95.

[23] Linsenmaier U, Wirth S, Reiser M, et al (2008) . Diagnosis and classification of pancreatic and duodenal injuries in emergency radiology. Radiographics; 28(6):1591-602.

[24] Hamdi Hameed Almaramhy, Salman Yousuf Guraya, (2012) . Computed tomography for pancreatic injuries in pediatric blunt abdominal trauma World J. Gastrointest Surg.4(7): 166170 .

[25] Jürgen K. Willmann, Justus E. Roos, Andreas Platz, et al ( 2002), Multidetector CT: Detection of Active Hemorrhage in Patients with Blunt Abdominal Trauma, (2) 179. 